\title{
Obtenção da farinha do resíduo do processamento de acerola e avaliação de compostos bioativos e nutritivos
}

\author{
Obtaining flour from acerola processing residue and evaluating bioactive and nutritive compounds \\ Obtención de harina a partir de residuos de procesamiento de acerola y evaluación de compuestos
}

bioactivos y nutritivos

Recebido: 16/09/2021 | Revisado: 29/09/2021 | Aceito: 27/10/2021 | Publicado: 30/10/2021

\author{
Marcela Paula Drumond Magalhães \\ ORCID: https://orcid.org/0000-0002-8635-025X \\ Universidade Federal de Ouro Preto, Brasil \\ E-mail: marcela-drumond@hotmail.com \\ Kelly Moreira Bezerra Gandra \\ ORCID: https://orcid.org/0000-0002-7934-9218 \\ Universidade Federal de Ouro Preto, Brasil \\ E-mail: kelly_gandra@yahoo.com.br \\ Luciana Rodrigues da Cunha \\ ORCID: https://orcid.org/0000-0001-5725-048X \\ Universidade Federal de Ouro Preto, Brasil \\ E-mail: lrcunha@ufop.edu.br \\ Emília Maria França Lima \\ ORCID: https://orcid.org/0000-0001-8768-4772 \\ Universidade Federal de Ouro Preto, Brasil \\ E-mail: emiliamflima@gmail.com
}

\begin{abstract}
Resumo
A acerola é uma fruta de grande valor nutricional reconhecida pelo elevado teor de vitamina $\mathrm{C}$, e possui variada aplicabilidade na indústria de alimentos. O seu processamento gera uma quantidade considerável de resíduos, e há carência de informações sobre a composição e o aproveitamento deste material. Diante do exposto, o objetivo desse estudo foi produzir uma farinha a partir do resíduo do despolpamento da acerola, e realizar a caracterização físicoquímica e análise de compostos bioativos, visando um possível aproveitamento para fins alimentícios. A farinha, constituída de semente e casca da fruta, mostrou-se um subproduto rico nutricionalmente, contendo $9,12 \%$ de proteína, 3,40\% de lipídios, $1,76 \%$ de cinzas, $6,64 \%$ de umidade e 79,07\% carboidratos, $\mathrm{pH}$ de 3,23 e acidez titulável de 3,30 g ácido cítrico/100g. Quanto aos compostos bioativos, a farinha apresentou elevados teores de vitamina $\mathrm{C}$ $(764,40 \mathrm{mg} / 100 \mathrm{~g})$, carotenoides $(23,93 \mu \mathrm{g} / \mathrm{g})$, compostos fenólicos totais $(28,46 \mathrm{mg}$ AGE/100 g), atividade antioxidante pelo radical DPPH (EC50 113,40 g de farinha/g DPPH) e ABTS (193,90 $\mu \mathrm{mol} / \mathrm{g}$ de farinha). A farinha desenvolvida apresentou-se como alternativa de aproveitamento para fins alimentícios, com boa conservação devido ao baixo teor de água, além de contribuir para a redução dos resíduos agroindustriais.
\end{abstract}

Palavras-chave: Carotenoides; Compostos bioativos; Composição centesimal; Malpighia ssp.; Subprodutos.

\begin{abstract}
Acerola is a fruit of great nutritional value recognized for its high vitamin $\mathrm{C}$ content, and has varied applicability in the food industry. Its processing generates a considerable amount of waste, and there is a lack of information on the composition and use of this material. This study aimed to produce a flour from the residue of acerola pulping, carry out the physicochemical characterization and analysis of bioactive compounds, aiming at a possible use for food purposes. The flour, consisting of seed and peel of the fruit was shown to be a nutritionally rich by-product, containing $9.12 \%$ protein, $3.40 \%$ lipids, $1.76 \%$ ash, $6.64 \%$ moisture and $79.07 \%$ carbohydrates, $\mathrm{pH}$ of 3.23 and titratable acidity of $3.30 \mathrm{~g}$ citric acid $/ 100 \mathrm{~g}$. As for bioactive compounds, the flour showed high levels of vitamin $\mathrm{C}$ $(764.40 \mathrm{mg} / 100 \mathrm{~g})$, carotenoids $(23.93 \mu \mathrm{g} / \mathrm{g})$, total phenolic compounds $(28.46 \mathrm{mg} \mathrm{AGE} / 100 \mathrm{~g})$, antioxidant activity by radical DPPH (EC50 $113.40 \mathrm{~g}$ of flour/g DPPH) and ABTS (193.90 $\mu \mathrm{mol} / \mathrm{g}$ of flour). The flour developed presented itself as an alternative to be used for food purposes, with good conservation due to its low water content, in addition to contributing to the reduction of agro-industrial residues.
\end{abstract}

Keywords: Carotenoids; Bioactive compounds; Centesimal composition; Malpighia ssp.; Byproducts.

\section{Resumen}

La acerola es una fruta de gran valor nutricional reconocida por su alto contenido en vitamina $\mathrm{C}$, y tiene variada aplicabilidad en la industria alimentaria. Su procesamiento genera una cantidad considerable de residuos, y existe una 
falta de información sobre la composición y uso de este material. El objetivo de este estudio fue producir una harina a partir del residuo de la pulpa de acerola, y realizar la caracterización fisicoquímica y análisis de compuestos bioactivos, con el objetivo de su posible uso con fines alimentarios. La harina, que consiste en la semilla y la cáscara de la fruta, demostró ser un subproducto rico en nutrientes, que contiene 9,12\% de proteína, 3,40\% de lípidos, 1,76\% de ceniza, 6,64\% de humedad y 79,07\% de carbohidratos, $\mathrm{pH}$ de 3,23 y acidez titulable de 3,30 g de ácido cítrico / 100 g. En cuanto a los compuestos bioactivos, la harina mostró altos niveles de vitamina C (764,40 mg / $100 \mathrm{~g})$, carotenoides $(23,93 \mu \mathrm{g} / \mathrm{g})$, compuestos fenólicos totales (28,46 mg AGE / $100 \mathrm{~g})$, actividad antioxidante por radical DPPH (EC50 113,40 g de harina / g DPPH) y ABTS (193,90 $\mu \mathrm{mol} / \mathrm{g}$ de harina). La harina desarrollada se presentó como una alternativa para su uso con fines alimentarios, con buena conservación por su bajo contenido en agua, además de contribuir a la reducción de residuos agroindustriales.

Palabras clave: Carotenoides; Compuestos bioactivos; Composición próxima; Malpighia ssp.; Subproductos.

\section{Introduçãa}

A fruta do gênero Malpighia ssp, popularmente conhecida como acerola, apresenta aroma frutado e doce quando madura, é utilizada como matéria prima para o processamento de sorvetes, geleias, compotas, iogurtes, sucos e mais comumente na forma de polpa, principalmente para o mercado externo (Antunes et al. 2013; Silva et al. 2016, Moura et al. 2016).

O Brasil destaca-se por ser um dos três maiores países produtores de frutas do mundo, com produção que supera 41 milhões de toneladas por ano (Batista et al. 2018; Rondan-Sanabria et al. 2019). A acerola passou a ter relevância na economia nacional quando o país se tornou o maior produtor, consumidor e exportador dessa fruta, com destaque para a região nordeste (Freitas et al. 2006; Dala-Paula et al. 2019). A acerola tornou-se popular como um alimento funcional entre os consumidores devido ao valor nutritivo e elevados teores de compostos fitoquímicos em sua composição, como o ácido ascórbico, compostos fenólicos e carotenoides (Reis et al. 2017; Rezende et al. 2018; Dala-Paula et al. 2019).

Os compostos fitoquímicos estão presentes em frutas e hortaliças e são amplamente estudados devido ao potencial benéfico à saúde. As substâncias antioxidantes provenientes da dieta auxiliam o mecanismo de defesa celular ao complexar com radicais livres e neutralizá-los, protegendo os componentes das células de alterações oxidativas, reduzindo assim, o risco de doenças (Batista et al. 2018; Seraglio et al. 2018; Sousa et al. 2020).

Os compostos fenólicos constituem uma importante classe dos fitoquímicos e são amplamente encontrados em frutas. São metabólitos secundários envolvidos na adaptação às condições de estresse e variam conforme aspectos genéticos, condições ambientais e grau de maturação da fruta (Sousa et al. 2011; Mariano-Nasser et al. 2017). Existem mais de 8000 estruturas conhecidas e, devido à atividade antimicrobiana, são pesquisadas para potenciais aplicações na conservação de alimentos (Carvalho et al. 2020; Santos et al. 2020). Os principais compostos fenólicos presentes na acerola são: rutina, quercetina, ácido cafeico, kaempferol e isoramnetina (Seraglio et al. 2018; Dala-Paula et al. 2019; Oliveira et al. 2020).

Os carotenoides são compostos bioativos que contribuem para o desenvolvimento embrionário e reprodução, modulação imunológica e manutenção do sistema visual (Müller-Maatsch et al. 2016). Além disso, conferem coloração vermelho-alaranjada a frutas (Comert et al. 2020), como na acerola.

$\mathrm{O}$ ácido ascórbico, precursor da vitamina $\mathrm{C}$, é conhecido como um importante composto antioxidante de origem natural. Está presente em diversas frutas, e a acerola é considerada um alimento funcional principalmente pelos altos teores dessa substância (Mariano-Nasser et al. 2017, Dala-Paula et al. 2019).

Enquanto essas propriedades benéficas estimulam o consumo de frutas com o objetivo de prevenir doenças (Batista et al. 2018), a industrialização permite um melhor aproveitamento das frutas, diversificando as possibilidades de comercialização, ampliando o tempo de conservação, a distribuição no mercado interno e externo e reduzindo as perdas pós-colheita (Reis et al. 2017; Seraglio et al. 2018). 
A industrialização da acerola gera toneladas de resíduos como sementes e bagaço, sendo necessário destinar adequadamente esse material, de modo que tenha menor impacto ao meio ambiente (Rezende et al. 2018). Uma alternativa para aproveitamento de resíduos agroindustriais é a transformação em farinha, visto que a desidratação é uma técnica de conservação capaz de garantir a estabilidade do produto por maior tempo (Silva et al. 2016, Reis et al. 2017). A farinha permite a utilização do subproduto na alimentação humana, sendo uma fonte potencial de nutrientes, como fibras, vitaminas, minerais e substâncias antioxidantes (Silva et al. 2016) e contribui para a elaboração de produtos com enriquecimento nutricional e valor agregado (Moura et al. 2016; Ferreira et al. 2020). Agregar valor a esses subprodutos agroindustriais é de interesse econômico e ambiental, pois pode auxiliar na redução dos impactos ambientais provenientes do descarte irregular dos resíduos (Ferreira et al. 2020, Oliveira et al. 2020).

Diante do exposto, o objetivo do presente estudo foi obter a farinha do resíduo do processamento de acerola e realizar a caracterização físico-química e avaliação de compostos bioativos, visando um aproveitamento do subproduto para fins alimentícios.

\section{Metodologia}

\subsection{Obtenção da farinha}

Inicialmente, as acerolas foram colhidas maduras na cidade de Itabirito, Minas Gerais. As frutas foram selecionadas, sanitizadas em solução de hipoclorito de sódio a 150 ppm e submetidas ao despolpamento (despolpadeira Braesi, DES-60, Brasil). A polpa de acerola foi utilizada em outro estudo. O resíduo obtido, composto de sementes e cascas, foi submetido à secagem em secador de bandejas com circulação forçada de ar (Polidryer, PD-25) a temperatura de $60 \pm 5{ }^{\circ} \mathrm{C}$, até atingir $6 \%$ de umidade, determinada por balança térmica por infravermelho (Eurotherm, Gibertini). O subproduto desidratado foi submetido à pulverização em moinho de pinos (Pulverisette 14, Fritsh Alemanha) e o material foi armazenado à temperatura ambiente em frascos de vidro, ao abrigo da luz.

\subsection{Caracterização físico-química}

A composição centesimal da farinha foi determinada de acordo com o Instituto Adolfo Lutz (2008), em triplicata. A umidade foi determinada por gravimetria em estufa a $105{ }^{\circ} \mathrm{C}$ (Balance Eletronic FA2100N Bioprecise, Brasil). A fração lipídica foi extraída pelo método de Sohxlet, utilizando éter de petróleo. As proteínas foram extraídas pelo sistema Kjeldahl, no qual foi feita a digestão de 0,25 g da amostra em bloco digestor (Gerhardt, Brasil), seguida de destilação (Technal TE-0363, Brasil), titulação com ácido clorídrico $0,1 \mathrm{~mol} / \mathrm{L}$, e fator de conversão de 6,25. As cinzas totais foram determinadas por gravimetria, após incineração de cerca de $1,0 \mathrm{~g}$ de amostra em mufla por $6 \mathrm{~h}$ à temperatura de $550{ }^{\circ} \mathrm{C}$. Os carboidratos foram estimados por diferença, subtraindo-se de $100 \%$ a soma dos valores obtidos para as determinações de umidade, lipídios, proteínas e cinzas.

Para aferição do $\mathrm{pH}$, pesou-se 5,0 g de amostra e diluiu-se em $50 \mathrm{~mL}$ de água destilada. Em seguida, realizou-se a medição com potenciômetro digital (Bel Engineering, W3B, Itália) previamente calibrado. A acidez total foi determinada por titulação com solução padronizada de $\mathrm{NaOH} 0,1 \mathrm{~mol} / \mathrm{L}$ e solução de fenolftaleína $1 \%$ como indicador, sendo o resultado expresso como ácido cítrico equivalente (Instituto Adolfo Lutz, 2008).

\subsection{Determinação dos compostos bioativos}

Inicialmente, preparou-se o extrato da farinha de acordo com Larrauri et al. (1997), com adaptações. Pesaram-se aproximadamente 5,0 $\mathrm{g}$ de amostras em erlenmeyers, aos quais foram adicionados 40,0 $\mathrm{mL}$ da solução metanol/água (50:50 $\mathrm{v} / \mathrm{v}$ ). A amostra foi mantida por $1 \mathrm{~h} \mathrm{em}$ agitador (ACBLabor, Brasil) a $25^{\circ} \mathrm{C}$ a $150 \mathrm{rpm}$. Os frascos foram armazenados em 
ambiente refrigerado $\left(8,0{ }^{\circ} \mathrm{C}\right)$ por $30 \mathrm{~min}$ para recuperação do sobrenadante. Em seguida, adicionaram-se $40 \mathrm{~mL}$ de acetona/água (70:30 v/v) ao resíduo, e após extração por $60 \mathrm{~min}$ com agitação de $150 \mathrm{rpm}$, foram mantidos em ambiente refrigerado $\left(8^{\circ} \mathrm{C}\right)$ por 30 min e o sobrenadante recuperado. $\mathrm{O}$ extrato obtido foi transferido para um balão volumétrico de 100 $\mathrm{mL}$ e adicionado de água destilada, sendo armazenados a $-20^{\circ} \mathrm{C}$. O extrato foi preparado ao abrigo da luz, bem como as análises de determinação dos compostos bioativos.

Os compostos fenólicos totais foram determinados pelo método adaptado de Waterhouse (2002). Foram adicionados 0,5 mL dos extratos em tubos de ensaio. Em seguida, adicionou-se 2,5 mL do reagente de Folin-Ciocalteu $10 \%$ (v/v) e 2,0 mL da solução de carbonato de sódio 4,0 \% (p/v). O homogenato foi mantido em repouso por $120 \mathrm{~min}$, seguido da leitura em espectrofotômetro (Global Trade Technology, Brasil) a $750 \mathrm{~nm}$. A determinação do teor de fenólicos totais foi realizada utilizando-se curva padrão de ácido gálico $(1,5,10,15,25$, e $50 \mu \mathrm{g} / \mathrm{mL})$. Os resultados foram expressos em mg ácido gálico equivalente por grama de amostra (mg AGE/g de amostra). Os resultados foram expressos em $\mu \mathrm{g}$ de ácido gálico equivalente (AGE)/g de farinha.

A atividade antioxidante, pelo radical ABTS (3-etilbenzotiazolina-6-ácido sulfônico), foi determinada de acordo com Rufino et al. (2007a). Adicionaram-se 30,0 $\mu \mathrm{L}$ dos extratos em tubos de ensaio e 3,0 mL do radical ABTS. Os tubos ficaram em repouso por 6 min, em seguida determinou-se a absorbância a $734 \mathrm{~nm}$. A determinação da atividade antioxidante foi obtida utilizando-se curva de calibração construída com padrões de trolox (100, 500, 1000, 1500 e $2000 \mu \mathrm{M})$. Os resultados foram expressos como $\mu \mathrm{mol}$ trolox/g de farinha.

A atividade antioxidante, pelo radical DPPH (2,2-difenil-1-picrilhidrazila), foi determinada de acordo com Rufino et al. (2007b). Alíquotas de $0,1 \mathrm{~mL}$ dos extratos foram adicionadas a 3,9 $\mathrm{mL}$ da solução de DPPH $(0,06 \mathrm{mM})$ e mantidas à temperatura ambiente por $120 \mathrm{~min}$. A leitura da absorbância foi determinada a $515 \mathrm{~nm}$. A curva padrão foi preparada com soluções de DPPH em diferentes concentrações $(10,20,30,40,50$ e $60 \mu \mathrm{M})$. Os resultados foram expressos em $\mathrm{EC}_{50}(\mathrm{~g}$ de farinha/g DPPH).

A determinação da vitamina $\mathrm{C}$ iniciou-se com a diluição da amostra em água destilada. Em seguida, adicionaram-se $10 \mathrm{~mL}$ de ácido sulfúrico $(20 \% \mathrm{v} / \mathrm{v}), 1 \mathrm{~mL}$ de solução de iodato de potássio $(10 \% \mathrm{~m} / \mathrm{v})$ e $1 \mathrm{~mL}$ de solução de amido $(1 \% \mathrm{~m} / \mathrm{v})$ como indicador. As amostras foram tituladas com solução de iodato de potássio $0,02 \mathrm{~mol} / \mathrm{L}$ até aparecimento da coloração acinzentada (Instituto Adolfo Lutz, 2008).

A extração de carotenoides foi realizada de acordo com Rodriguez-Amaya (2001). Em 5,0 g das amostras foram adicionados $40 \mathrm{~mL}$ de acetona e procedeu-se à agitação (ACBLabor, Brasil) da mistura por $1 \mathrm{~h}$ a $150 \mathrm{rpm}$. Em seguida, procedeu-se a filtragem a vácuo e cada amostra foi lavada com acetona por três vezes. A um funil de separação, foram adicionados $45 \mathrm{~mL}$ de éter de petróleo e os pigmentos foram transferidos, em pequenas frações, seguidas de água destilada, descartando-se a fase inferior. As amostras foram lavadas com água destilada por quatro vezes. A solução dos pigmentos em éter de petróleo foi transferida para um balão volumétrico de $100 \mathrm{~mL}$ com éter de petróleo. Em seguida, foram feitas as leituras da absorbância (Global Trade Technology, Brasil), em comprimentos de onda específicos para cada carotenoide (449 nm para $\beta$-criptoxantina, $450 \mathrm{~nm}$ para $\beta$-caroteno e $470 \mathrm{~nm}$ para licopeno). Para a determinação dos carotenoides totais, foi considerado o maior pico de absorbância obtido nas leituras (Carvalho et al. 2015), sendo detectado a $449 \mathrm{~nm}$. O teor de carotenoides totais foi obtido pela equação 01 .

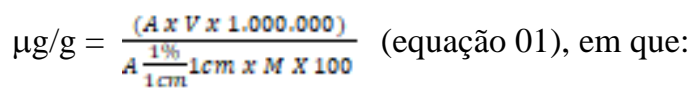

$\mathrm{A}=$ absorbância da solução; $\mathrm{V}=$ volume final da solução $(\mathrm{mL}) ; \mathrm{A}{ }^{1 \%}{ }_{1 \mathrm{~cm}}=$ coeficiente de absortividade molar do pigmento em éter de petróleo (Rodriguez-Amaya 2001); M = massa da amostra tomada para a análise (g). 


\section{Resultados e Discussão}

\subsection{Caracterização físico-química da farinha}

A composição centesimal de um alimento exprime a proporção em que aparecem em $100 \mathrm{~g}$ da porção comestível do produto, grupos homogêneos de substâncias que o constituem. Assim, é determinado o valor nutritivo do alimento. A Tabela 1 apresenta os valores médios dos resultados das análises físico-químicas obtidas para a farinha de resíduos de acerola.

Tabela 1: Caracterização físico-química da farinha de resíduo de acerola.

\begin{tabular}{ll}
\hline Análises físico-químicas & Média \pm DP* \\
\hline Umidade (\%) & $6,64 \pm 0,51$ \\
Cinzas (\%) & $1,76 \pm 0,04$ \\
Proteínas (\%) & $9,12 \pm 0,18$ \\
Lipídios (\%) & $3,40 \pm 0,32$ \\
Carboidratos (\%) & $79,07 \pm 0,03$ \\
Acidez titulável (g ácido cítrico/100g) & $3,30 \pm 0,23$ \\
$\mathrm{pH}$ & $3,23 \pm 0,01$ \\
\hline
\end{tabular}

*DP: desvio padrão. Análises realizadas em triplicata.

Fonte: Autores (2021).

A redução do teor de umidade é um fator importante para a conservação de alimentos e ajuda a aumentar a vida útil do produto. A umidade da farinha em estudo foi de 6,64\% (Tabela 1), o que caracteriza um produto com baixa umidade e alta durabilidade. O valor encontrado foi superior ao encontrado por Ferreira et al. (2020) para farinha de semente de abóbora $(5,25 \%)$ e inferior ao encontrado por Aquino et al. (2010) para a farinha do resíduo de acerola (8,60\%). Todos os resultados enquadram-se na legislação nacional, que estabelece que os teores de umidade de farinhas não ultrapassem 15\% (Brasil, 2005).

O teor de cinzas em alimentos refere-se ao resíduo inorgânico remanescente da queima de matéria orgânica a elevadas temperaturas. Portanto, as cinzas indicam a presença de minerais no alimento, o que implica em seu valor nutricional, visto que os minerais são importantes para a saúde e essenciais para o organismo. A determinação das cinzas também é importante por outras razões: quando o valor encontrado é consideravelmente superior ao esperado, pode indicar uma possível fraude no alimento, como por exemplo, a adição de areias em farinhas para aumentar o rendimento. Além disso, as cinzas podem ser o ponto de partida para identificação de minerais específicos (Cosmo; Galeriani, 2017). O teor de cinzas encontrado no presente estudo (1,76\%) foi superior ao valor observado por Aguiar (2010) para a farinha de semente de acerola $(0,44 \%)$ e inferior aos valores encontrados por Sobrinho (2014) para o mesmo subproduto (4,87\% a 5,58\%). A quantidade de matéria inorgânica presente, quantidade de água da fruta (Sobrinho 2014), composição do solo e condições de cultivo são fatores que podem interferir na presença de minerais nos alimentos (Cecchi 2007).

O teor de proteína encontrado no presente estudo $(9,12 \%)$ foi similar ao encontrado por Borges $(2011)(9,45 \%)$, Sobrinho (2014) $(6,11 \%$ a 8,70\%) e Soares et al. (2001) (9,12\%) para a farinha do resíduo de acerola, e para a polpa da fruta em pó $(9,05 \%)$ (Soares et al 2001), indicando similaridade do teor proteico tanto na fruta quanto no resíduo. Esses dados evidenciam a possibilidade de utilizar a farinha do resíduo de acerola como complemento para formulações alimentícias, no intuito de agregar valor proteico. Neste sentido, o estudo realizado por Anjos et al. (2017) mostrou que a adição de farinha de semente de abóbora em pães sem glúten aumentou o teor de proteínas, lipídios e fibras dos pães, mostrando-se como alternativa para aplicação em produtos de panificação, proporcionando um produto rico nutricionalmente. 
Em relação à fração lipídica, o resultado encontrado (3,40\%) está próximo ao encontrado por Storck et al. (2015) para farinha de outras frutas, como laranja (4,19\%) e maçã (3,02\%). O baixo teor de lipídios no resíduo de frutos é esperado, uma vez que o lipídio é um dos componentes de menor proporção em frutos, com exceção do abacate que possui uma elevada quantidade de óleo (Cecchi 2007).

O teor de carboidrato encontrado no presente estudo $(79,07 \%)$ corrobora o resultado encontrado por Pereira et al. (2013) para o mesmo subproduto $(81,29 \%)$, o que caracteriza a farinha do resíduo de acerola com elevado teor de carboidrato. Borges et al. (2021) encontraram valor próximo $(81,41 \%)$ em farinha de resíduo de açaí, e discutem que valores elevados de carboidratos se devem às macromoléculas, como celulose e amido. Além dessas, pectina e outros biopolímeros também estão presentes em cascas de vegetais, e as fibras estão incluídas no valor encontrado para carboidratos.

Assim como a atividade de água, o baixo $\mathrm{pH}$ também é um fator importante para a conservação dos alimentos. $\mathrm{O}$ pH encontrado na presente farinha $(3,23)$ está próximo ao encontrado por Pereira et al. $(2013)(3,69)$ e Sobrinho $(2014)(2,73$ a 4,02) para o mesmo subproduto, valores já esperados para frutas in natura. Sendo o pH de 4,5 limitante para o desenvolvimento de microrganismos, estas farinhas podem ser consideradas ácidas com baixo ou difícil crescimento de bactérias (Sobrinho 2014).

Além da atividade de água e $\mathrm{pH}$, a acidez também é importante parâmetro na determinação da conservação do alimento, visto que a acidificação possui ação inibidora do crescimento microbiano (Freitas 2015). Na farinha em estudo, a acidez titulável $(3,30 \%)$ foi inferior à encontrada na farinha do mesmo subproduto (4,68\%) por Pereira et al. (2013), enquanto Freitas et al. (2015) observaram valores entre 5,29 a 12,10\%, para farinhas de resíduos do processamento de morango e cupuaçu, respectivamente. Em farinhas de frutas, a acidez tende a aumentar significativamente com o tempo de armazenamento devido a mudanças químicas que podem ocorrer no produto (Reis et al. 2017), fato que pode estar relacionado com os diferentes valores observados nestes estudos. Para evitar alterações nas características sensoriais, é recomendado o armazenamento sob refrigeração (Freitas et al. 2015).

Vários fatores influenciam na composição físico-química de frutas, como a espécie, o estádio de maturação, época de colheita, condições de estresse como escassez de nutrientes, e condições ambientais como disponibilidade hídrica e luminosidade (Rondan-Sanabria et al. 2019, Seraglio et al. 2018). A influência desses fatores explica as diferenças entre os resultados obtidos nos diferentes estudos.

\subsection{Determinação dos compostos bioativos na farinha}

A acerola é uma fruta que possui elevado teor de compostos bioativos, como compostos fenólicos, carotenoides e ácido ascórbico (Mariano-Nasser et al. 2017; Rezende et al. 2018; Dala-Paula et al. 2019). Com o despolpamento da fruta e transformação do resíduo em farinha, alguns compostos são eliminados, visto a exposição à luz, oxigênio, calor e demais fatores que degradam estes compostos. No entanto, a farinha obtida neste estudo apresentou valores consideráveis, mesmo após os procedimentos, conforme demonstrado na Tabela 2. 
Tabela 2: Compostos bioativos na farinha do resíduo do despolpamento de acerola.

\begin{tabular}{lc}
\hline Compostos bioativos & Média \pm DP* \\
\hline Atividade antioxidante - ABTS $(\mu \mathrm{mol} / \mathrm{g}$ de farinha) & $193,90 \pm 17,22$ \\
Atividade antioxidante - DPPH (EC50 - g de farinha/g DPPH) & $113,40 \pm 7,87$ \\
Compostos fenólicos totais $(\mathrm{mg}$ AGE/100g) & $28,46 \pm 3,90$ \\
Carotenoides $(\mu \mathrm{g} / \mathrm{g})$ & $23,93 \pm 0,13$ \\
Vitamina C $(\mathrm{mg} / 100 \mathrm{~g})$ & $764,40 \pm 69,60$ \\
\hline
\end{tabular}

*DP: desvio padrão. Análises realizadas em triplicata.

Fonte: Autores (2021).

A avaliação e o consumo de compostos com atividade antioxidante vêm atraindo interesse de pesquisadores e consumidores, por estarem associados à diminuição de doenças degenerativas, oriundas da presença de radicais livres. A atividade antioxidante é atribuída à capacidade de extinguir os radicais livres, doando átomos de hidrogênio, elétrons ou cátions metálicos quelantes. Dessa forma, são capazes de prevenir os efeitos deletérios da oxidação e, quando incorporados à dieta humana, promovem benefícios à saúde (Mariano-Nasser et al. 2017; Chuah et al. 2020). Os efeitos benéficos dos antioxidantes naturais estão relacionados à presença de ácido ascórbico, antocianinas, carotenoides, compostos fenólicos e outros com atividade antioxidante, que podem ser encontrados em frutas e vegetais (Rufino et. al. 2007; Batista et al. 2018; Carvalho et al. 2020).

$\mathrm{Na}$ farinha em estudo, a atividade antioxidante com o radical ABTS (193,90 $\mu$ mol Trolox/g) foi relativamente inferior ao resultado encontrado por Sena et al. (2014) para farinha do resíduo da acerola $(247,24 \mu \mathrm{M}$ Trolox/g). Essas diferenças já são esperadas, devido a diversos fatores que podem influenciar na atividade antioxidante dos frutos, como a espécie, o estado de maturação, maior tempo de armazenamento e exposição da fruta ou do resíduo à luz, que podem degradar os compostos antioxidantes (Soares et al. 2008).

O potencial do extrato da farinha para sequestrar radicais livres DPPH foi expresso em EC50, e o valor encontrado é inversamente relacionado à atividade antioxidante. Um extrato que apresenta alto potencial em sequestrar radicais livres possui baixo valor de EC50, de modo que uma pequena quantidade do extrato é capaz de reduzir a concentração inicial do radical DPPH em 50\% (Rufino et al. 2007b). A farinha em estudo apresentou atividade antioxidante similar ao resultado encontrado por Pereira et al. (2013) para farinha de resíduos de acerola (88,38 EC50 - g de farinha/g DPPH).

O teor de compostos fenólicos totais na farinha em estudo foi inferior aos valores encontrados por Sobrinho (2014), também para a farinha do resíduo de acerola (191,5 mg AGE/100g). Pelo fato desses compostos pertencerem ao metabolismo secundário das plantas, os teores são influenciados pelas condições ambientais e de estresse da planta, como a maturação, origem geográfica, condições de colheita e armazenamento, além da peculiaridade metodológica relacionada ao solvente extrator (Soares et al. 2008; Rais et al. 2019) e o processamento da farinha. Esses fatores explicam as diferenças existentes entre compostos bioativos para uma mesma fruta.

O teor de carotenoides totais $(23,93 \mu \mathrm{g} / \mathrm{g})$ foi superior ao valor encontrado por Aquino et. al (2010) na caracterização físico-química em farinha do resíduo da acerola $(8,09 \mu \mathrm{g} / \mathrm{g})$.

Com relação ao ácido ascórbico, a farinha apresentou um resultado relevante $(764,40 \mathrm{mg} / 100 \mathrm{~g})$, quando comparado com os padrões de identidade e qualidade de polpas de frutas, que determina que o teor de vitamina C em polpa de acerola seja no mínimo 800,0 mg/100g (Brasil, 2000). Em se tratando da farinha do resíduo do processamento da fruta, este é um resultado interessante, visto que o teor de vitamina C encontrado assemelha-se ao valor recomendado para a polpa da fruta. Assim, destaca-se o potencial da farinha em estudo para aplicação e enriquecimento de alimentos. 
O desenvolvimento de produtos alimentícios acrescidos de farinhas de cascas e sementes vem sendo estudado recentemente, como uma estratégia que contribui com a redução do desperdício de alimentos e que agrega valor nutricional na criação de novas formulações. Alguns exemplos de produtos bem avaliados nos testes de aceitação e/ou intenção de compra são os pães sem glúten adicionados de farinha de resíduos de abóbora desenvolvidos por Anjos et al. (2017), os pães de forma integral com farinha do resíduo de mamão desenvolvidos por Santos et al. (2018) e os quibes adicionados de farinha de açaí desenvolvidos por Borges et al. (2021).

Com relação às propriedades tecnológicas, a adição das farinhas em grande quantidade pode prejudicar algumas características dos produtos de panificação, por comprometer a formação da rede de glúten, como a circularidade dos alvéolos (Ferreira et al., 2020) e o aumento da densidade (Santos et al., 2018). Por outro lado, essas farinhas possuem valores consideráveis de compostos bioativos com atividade antioxidante, que podem atuar como conservantes, retardando a oxidação. Atualmente, os consumidores prezam pela qualidade do produto com ingredientes naturais, e os antioxidantes naturalmente presentes constituem uma possibilidade de substituição dos sintéticos, agregando valor ao produto final (Lima et al., 2019).

\section{Conclusão}

A farinha obtida do resíduo do despolpamento de acerola mostrou-se uma alternativa de aproveitamento para fins alimentícios, uma vez que apresentou baixo teor de umidade, conferindo elevado tempo de conservação, e considerável valor nutricional, com destaque para o teor de proteínas e carboidratos, ácido ascórbico e atividade antioxidante. Além disso, o processamento da farinha pode diminuir a quantidade de resíduos produzidos, reduzindo os impactos ambientais.

Sugere-se, antes da incorporação na dieta humana, a avaliação da biodisponibilidade dos nutrientes, presença de fatores antinutricionais e potenciais alergênicos, e futuramente, o desenvolvimento de novos produtos com valor agregado, como por exemplo, em produtos de panificação.

\section{Referências}

Aguiar, T. M., Rodrigues, F. S., Santos, E. R. \& Sabaa-Srur, A. U. O. (2010). Chemical characterization and evaluation of the nutritional value of Malpighia punicifolia seeds. Nutrire, 35(2), 91-102.

Anjos, C.N., Barros, B. H.S., Silva, E.I.G., Mendes, M. L. M., \& Messias, C. M. B. O. (2017). Desenvolvimento e aceitação de pães sem glúten com farinhas de resíduos de abóbora (Cucurbita moschata). Arquivos de Ciências da Saúde, 24(4), 58-62.

Antunes, A. E. C., Liserre, A. M., Coelho, A. L. A., Menezes, C. R., Moreno, I., Yotsuyanagi, K. \& Azambuja, N. C. (2013). Acerola nectar with added microencapsulated probiotic. LWT-Food Science and Technology, 54(1), 125-131.

Aquino, A. \& Leão, K. (2010). Obtenção e caracterização físico-química da farinha de resíduos do processamento de polpa de acerola. Revista Instituto Adolfo Lutz, 69(3), 379-86.

Batista, P. F., Lima, M. A. C., Alves, R. E. \& Façanha, R. V. (2018). Bioactive compounds and antioxidant activity in tropical fruits grown in the lowermiddle São Francisco Valley. Revista Ciência Agronômica, 49(4), 616-623.

BRASIL. Ministério da Agricultura e do Abastecimento. Instrução Normativa N$^{\circ} 1$, de 7 de janeiro de 2000 . Regulamento técnico geral para fixação dos padrões de identidade e qualidade para polpa de frutas. Diário Oficial da União, nº 6, Brasília, 2000.

BRASIL. Ministério da Agricultura, Pecuária e Abastecimento. Instrução Normativa no 8 , de 2 de junho de 2005 . Regulamento técnico de identidade e qualidade da farinha de trigo. Diário Oficial da União, n. 105, p. 91, 2005.

Borges, K. C. (2011). Estudo das características físico-químicas e funcionalidade de bagaços de frutas tropicais desidratadas em leito de jorro. Dissertação de mestrado - Universidade Federal do Rio Grande do Norte.

Borges, M. V., Sousa, E. B., Silveira, M. F. A., Souza, A. R. M., Alves, V. M., Nunes, L. B. M. \& Barros, S. K.A. (2021). Propriedades físico-químicas e tecnológicas da farinha do resíduo de açaí e sua utilização. Research, Society and Development, 10(5), e17810514517.

Carvalho, A. V., Mattietto, R. A., Rios, A. O., Maciel, R. A., Moresco, K. S. \& Oliveira, T. C. S. (2015). Bioactive compounds and antioxidant activity of pepper (Capsicum sp.) genotypes. Journal of Food Science and Technology, 52(11), 7457-7464.

Carvalho, F. M., Martins, J. T. A., Lima, E. M. F., Santos, H. V., Pereira, P. A. P., Pinto, U. M. \& Cunha, L. R. (2020). Pitanga and grumixama extracts: antioxidant and antimicrobial activities and incorporation into cellulosic films against Staphylococcus aureus. Research, Society and Development, 9(11), e1759119362. 
Cecchi, H. M. (2007). Fundamentos teóricos e práticos em análise de alimentos. Editora da UNICAMP.

Chuah, P.N., Nyanasegaram, D., Yu, K-X., Razik, R. M., Al-Dhali, S., Kue, C. S., Shaari, K. \& Ng, C. H. (2020). Comparative conventional extraction methods of ethanolic extracts of Clinacanthus nutans leaves on antioxidant activity and toxicity. British Food Journal, 122(10), 3139-3149.

Cömert, E. D., Mogol, B. A. \& Gökmen, V. (2020). Relationship between color and antioxidant capacity of fruits and vegetables. Current Research in Food Science, 2, 1-10.

Cosmo, B. M. C. \& Galeriani, T. M. (2017). Determinação de cinzas em amostras de beterraba, capim elefante e farinha de peixe. Revista Científica Semana Acadêmica, 01(113), 1-19.

Dala-Paula, B. M., Santos, T. P., Araújo, L. S., Bastos, R. R. A., Moraes, J. O. \& Carbonera, N. (2019). Domestic processing and storage on the physicalchemical characteristics of acerola juice (Malpighia glabra L.). Ciência e Agrotecnologia, 43, e021519.

Ferreira, C. M., Lima, S. B., Zambelli, R. A. \& Afonso, M. R. A. (2020). Effect of mixed flour from vegetable by-product on breads. Brazilian Journal of Development, 6(2), 8710-8724.

Freitas, C. A. S., Maia, G. A., Costa. J. M. C., Figueiredo, R. W. \& Souza, P. H. M. (2006). Acerola: production, composition, nutritional aspects and products. Current Agricultural Science and Technology, 12(4), 395-400.

Freitas, E. C., Barreto, E. S., Barros, H. E. A., Silva, A. C. M. \& Silva, M. V. (2015). Processamento e caracterização físico-química de farinhas de resíduos de polpas de frutas congeladas da Theobroma grandiflorum e Fragaria vesca. Revista Brasileira de Produtos Agroindustriais, 17(4), 425-432.

Instituto Adolfo Lutz. (2008). Métodos físico-químicos para análise de alimentos. (4a ed.), 533 p.

Larrauri, J. A., Rupérez, P. \& Saura-Calixto, F. (1997). Effect of drying temperature on the stability of polyphenols and antioxidant activity of red grape pomace peels. Journal of Agricultural and Food Chemistry, 45, 1390-1393.

Lima, E. M. F., Madalão, M. C. M., Santos Jr, W. C., Bernardes, P. C., Saraiva, S. H. \& Silva, P. I. (2019). Spray-dried microcapsules of anthocyanin-rich extracts from Euterpe edulis M. as an alternative for maintaining color and bioactive compounds in dairy beverages. Journal of Food Science and Technology, $56(9), 4147-4157$

Moura, A. A. C., Aroucha, E. M. M., Góis, V. A., Leite, R. H. L., Ferreira, R. M. A. \& Silva, M. C. P. (2016). Iogurtes com polpa de noni e acerola: avaliação físico-química, atividade antioxidante e perfil sensorial. Boletim do Centro de Pesquisa de Processamento de Alimentos, 34(2).

Mariano-Nasser, F. A. C., Nasser, M. D., Furlaneto, K. A., Ramos, J. Á., Vieites, R. L., Pagliarini, M. K. (2017). Bioactive compounds in different acerola fruit cultivares. Semina: Ciências agrárias, 38(4), 2505-2514.

Müller-Maatsch, J., Sprenger, J., Hempel, J., Kreiser, F., Carle, R. \& Schweiggert, R. M. (2017). Carotenoids from gac fruit aril (Momordica cochinchinensis [Lour.] Spreng.) are more bioaccessible than those from carrot root and tomato fruit. Food Research International, 99, 928-935.

Pereira, C.T.M., Silva, C. R. P., Lima, A., Pereira, D. M., Costa, C. N. \& Neto, A. A. C. (2013). Obtenção, caracterização físico-química e avaliação da capacidade antioxidante in vitro da farinha resíduo de acerola (Malpighia glabra L.) Acta tecnológica, 8(2), 50-56.

Oliveira, L. M. N., Silva, L. M. R., Lima, A. C. S., Almeida, R. R., Ricardo, N. M. P. S., Figueiredo, E. A. T. \& Figueiredo, R. W. (2020). Characterization of rutin, phenolic compounds and antioxidant capacity of pulps and by-products of tropical fruits. Research, Society and Development, 9(4), e42942812.

Rais, C., Driouch, A., Slimani, C., Bessi, A., Balouiri, M., El Ghadraoui, L., Lazraq, A. \& Figuigui, J. A. (2019). Antimicrobial and antioxidant activity of pulp extracts from three populations of Ziziphus lotus L. Nutrition \& Food Science, 49(6), 1014-1028.

Reis, D. S., Neto, A. F., Ferraz, A. V. \& Freitas, S. T. (2017). Production and storage stability of acerola flour dehydrated at different temperatures. Brazilian Journal of Food Technology, 20, e2015083.

Rezende, Y. R. R. S., Nogueira, J. P. \& Narain, N. (2018). Microencapsulation of extracts of bioactive compounds obtained from acerola (Malpighia emarginata DC) pulp and residue by spray and freeze drying: Chemical, morphological and chemometric characterization. Food Chemistry, $254,281-291$.

Rodriguez-Amaya, D. B. (2001). A guide to carotenoid analysis in food. Washington: International Life Sciences Institute. 64 p.

Rondan-Sanabria, G. G., Garcia, A. J. C., Montaño, H. S. P., Arias, E. C. S. \& Narain, N. (2019). Volatile compounds of da acerola (Malpighia emarginata) obtained by HS-SPME at two ripening stages. Revista de Ciências Agrárias (Portugal), 42(1), 266-274.

Rufino $^{a}$, M. S. M., Alves, R. E., Brito, E. S., Morais, S. M., Sampaio, C. G., Pérez-Jiménez, J. \& Saura-Calixto, F. D. (2007). Metodologia científica: determinação da atividade antioxidante total em frutas pela captura do radical livre ABTS. Comunicado Técnico - EMBRAPA Ceará.

Rufino $^{\text {, }}$ M. S. M., Alves, R. E., Brito, E. S., Morais, S. M., Sampaio, C. G., Pérez-Jiménez, J. \& Saura-Calixto, F. D. (2007). Metodologia científica: determinação da atividade antioxidante total em frutas pela captura do radical livre DPPH. Comunicado Técnico - EMBRAPA Ceará.

Santos, C. M. D., Rocha, D. A., Madeira, R. A. V., Queiroz, E. D. R., Mendonça, M. M., Pereira, J. \& Abreu, C. M. P. D. (2018). Preparação, caracterização e análise sensorial de pão integral enriquecido com farinha de subprodutos do mamão. Brazilian Journal of Food Technology, 21 , e2017120.

Santos, C. A., Almeida, F. A., Quecan, B. X. V., Pereira, P. A. P., Gandra, K. M. B., Cunha, L. R. \& Pinto, U. M. (2020). Bioactive properties of Syzygium cumini (L.) skeels pulp and seed phenolic extracts. Frontiers in Microbiology, 11:990.

Sena, D. N., Sousa, M. M. A., Sousa, P. H. M. \& Almeida, M. M. B. (2014). Estudo do potencial antioxidante em amostras de farinha de resíduos de processamento de acerola, tangerina e graviola. Anais do XX Congresso Brasileiro de Engenharia Química. 
Research, Society and Development, v. 10, n. 14, e188101420714, 2021

(CC BY 4.0) | ISSN 2525-3409 | DOI: http://dx.doi.org/10.33448/rsd-v10i14.20714

Seraglio, S. K. T., Schulz, M., Nehring, P., Betta, F. D., Valese, A. C., Daguer, H., Gonzaga, L. V, Fett, R. \& Costa, A. C. O. (2018). Determinação de compostos fenólicos por LC-MS/MS e capacidade antioxidante de acerola em três estádios de maturação comestíveis. Revista do Congresso Sul Brasileiro de Engenharia de Alimentos, 4(1), 96-110.

Silva, P. B., Duarte, C. R. \& Barrozo, M. A. S. (2016). Dehydration of acerola (Malpighia emarginata DC) residue in a new designed rotary dryer: Effect of process variables on main bioactive compounds. Food and Bioproducts Processing, 98, $62-70$.

Soares, E., Oliveira, G. S. F., Maia, G. A., Monteiro, J. C. S., Silva Jr., A. \& Filho, M. S. S. (2001). Desidratação da polpa de acerola (Malpighia emarginata d.c.) pelo processo "foam-mat". Ciência e Tecnologia de Alimentos, 21(2), 164-170.

Soares, M., Welter, L., Kuskoski, E. M., Gonzaga, L. \& Fett, R. (2008). Compostos fenólicos e atividade antioxidante da casca de uvas Niágara e Isabel. Revista Brasileira de Fruticultura, 30(1), 59-64.

Sobrinho, I. S. B. (2014). Propriedades nutricionais e funcionais e resíduos de abacaxi, acerola e cajá oriundos da indústria produtora de polpas. Dissertação de mestrado - Universidade Estadual do Sudoeste da Bahia.

Sousa, Y. A., Borges, M. A., Viana, A. F. S., Dias, A. L, Sousa, J. J. V., Silva, B. A., Silva, S. K. R. \& Aguiar, F. S. (2020). Physicochemical and microbiological assessment of frozen pulp marketed in Santarém - PA. Brazilian Journal of Food Technology, 23, e2018025.

Sousa, M. S. B., Vieira, L. M. \& Lima, A. (2011). Fenólicos totais e capacidade antioxidante in vitro de resíduos de polpas de frutas tropicais. Revista Brasileira de Fruticultura, v. 33(3).

Storck, C. R., Basso, C., Favarin, F. R. \& Rodrigues, A. C. (2015). Microbial quality and composition of flour from fruit juice production residues with different granulometries. Brazilian Journal of Food Technology, 18(4), 277-284.

Waterhouse, A. L. (2002). Polyphenolics: Determination of total phenolics. In: WROLSTAD, R. E. Current Protocols in Food Analytical Chemistry, 11, 111118. 